(C) 1993 IEEE. Personal use of this material is permitted. However, permission to reprint/republish this material for advertising or promotional purposes or for creating new collective works for resale or redistribution to servers or lists, or to reuse any copyrighted component of this work in other works must be obtained from the IEEE.

\title{
Progress of the LEP Energy Upgrade Project
}

\author{
Carlo Wyss \\ CERN-AC \\ $\mathrm{CH}-1211$ Geneva 23
}

\begin{abstract}
Beams approaching $90 \mathrm{GeV}$ are expected in LEP in 1995; to this end, 192 superconducting accelerating cavities will be installed. The LEP energy upgrade also requires a low-emittance optics, layout modifications, new machine components, powerful cryoplants and a general upgrade of the LEP infrastructure, which will have to distribute and cool away about twice the present energy requirements. The programme was launched in December 1989 and by now major milestones have been achieved. The progress achieved in the various activities are presented, with the aim of providing a view of the programme goals and status.
\end{abstract}

\section{INTRODUCTION}

The LEP collider has been designed [1] to allow its upgrade from an initial energy of about $50 \mathrm{GeV}$ per beam (Phase 1) to an optimum energy near to $90 \mathrm{GeV}$, the ultimate limit being set at $125 \mathrm{GeV}$ by the magnet system. To run LEP at high energy in an economical way, $352 \mathrm{MHz}$ SC cavities (SCC's) have been developed [2] since the beginning of the LEP design. Various schemes [3] for the energy upgrading by adding SCC's were worked out; the one retained consists of 32 SCC's added to each of the LEP Pts 2 and 6, where the copper cavities are presently installed, and of the installation of 64 SCC's at each of the Pts 4 and 8 , where new RF accelerating stations are being created. A pilot project for the installation of a first set of 32 SCC's at the LEP Point 2 was launched in 1988. In December 1989 a programme [4] termed LEP 200 was started, aiming at upgrading the beam energy above the $\mathrm{W}$ pair production threshold.

\section{OPTICS AND LATTICE MODIFICATIONS}

A low-emittance optics, achieved by increasing the phase advance in the arcs from $60^{\circ}$ to $90^{\circ}$, is necessary to maximisc luminosity by approaching the beam-beam limit. In this way a luminosity of about $1.110^{31} \mathrm{~cm}-2 \mathrm{~s} 1$ is expected around 87 to $90 \mathrm{GeV}$, assuming $0.5 \mathrm{~mA} /$ bunch and 4 bunches per beam. Such an optics [5] has successfully been used for regular LEP operation during 1992. Furthermore the $90^{\circ}$ optics is also well suited for the 8-bunch scheme ("pretzel" scheme) also commissioned in 1992 [6], aiming at increasing luminosity by a factor two.

A number of geometry modifications of the original LEP lattice are necessary at the even and odd points of LEP, because of quadrupole magnets running out of focusing strength above $65 \mathrm{GeV}$ and because of an increase in length of the SC RF cells, due to the longer length of the SCC's assemblies. All the relevant studies [7] have been completed, the layout of the odd
IP's has already been modified during the $1992 / 1993$ winter shutdown.

\section{THE SC ACCELERATING SYSTEM}

\subsection{SC cavities}

The SCC's [8] are installed in LEP as 4-units modules, $11.285 \mathrm{~m}$ long. The first set of 32 SCC's, at Pt2, consists of eight prototype $\mathrm{Nb}$ sputtered cavities (two modules) made at CERN and $24 \mathrm{Nb}$ sheet cavities (six modules), out of which four are prototypes made in 1989 , and 20 are series units, delivered by industry during the years 1991 and 1992. For these cavities, a nominal gradient of $5 \mathrm{MV} / \mathrm{m}$ with a quality factor of $310^{9}$ was specified.

This performance has been achieved and exceeded by the $\mathrm{Nb}$ sputtered prototype units and by all the 20 series $\mathrm{Nb}$ sheet SCC's, the last of which has been accepted by October $1992(7 \mathrm{MV} / \mathrm{m}$ have consistently been attained with $Q$ values between 2 and $310^{9}$ ). The three SCC prototype modules operated in LEP during 1991 could not be fully conditioned to nominal performance before their installation because of difficulties that were later traced back to insufficient conditioning of the power couplers. In LEP they were run with beams up to an average of $3.7 \mathrm{MV} / \mathrm{m}$, delivering a total voltage of up to $76 \mathrm{MV}$. Uneven excitation of the four SCC's of a module, because of geometry tolerances, has been overcome by the design of an adjustable power coupler, required anyhow for optimum coupling at higher beam intensities. The RF conditioning of the main couplers (MC's) has turned out to be a key step, among others, in view of achieving the specified performances. To ensure efficient and safe conditioning, the MC's have been fitted with diagnostics like vacuum gauges and electron pick-up antennas. Mastery of contamination during assembly is as another key issue; contamination monitoring techniques as used for the production of VLSI circuits are at present being used at CERN to gain a deeper insight in this matter. In view of gaining experience and introducing the necessary modifications, the assembly at CERN of the series Nb sheet SCC modules has been slowed down; the first module of this kind, driven at $5 \mathrm{MV} / \mathrm{m}$ for 12 hours during lab tests, has been installed at LEP Pt 2 this April.

LEP Pts 6,8 and 4 will be equipped with $\mathrm{Nb}$ sputtered SCC modules, to be delivered fully assembled by industry. A nominal gradient of $6 \mathrm{MV} / \mathrm{m}$ with a quality factor of $410^{9}$ has been specified for this type of SCC's. At the time of writing 29 SC bare cavities $(17 \%$ of the total quantity) fulfilling the specifications, have been delivered by the three manufacturers, to which SCC's and module delivery were entrusted by contract in October 1990. After the 
acceptance test at CERN, the bare SCC's are shipped back to the manufacturers', for assembly into modules. The measurement of the first module delivered by industry shows that nominal performance has been achieved by two SCC's, the other two being slightly below, but that some contamination and irregular patterns in the LHe distribution to the HOM couplers prevent the whole module from being conditioned to nominal performance. These difficulties are being corrected and there is confidence that by September 1993 two Nb-film modules will be installed at LEP Pt6.

The series production of SCC units is now taking off and it is expected to be completed by end 1994; provided that the present difficulties with SCC's modules are solved in the next months, it is hoped to nearly complete during the 93-94 winter shutdown the installation of SCC modules at Pts 2 and 6, and possibly install a few modules at least at Pt 8.

\subsection{RF power and control}

Twelve new RF units are required for the 192 SCC's. Each unit consists of a $1.3 \mathrm{MW}$ klystron and circulator, the waveguide distribution system and 22 racks of electronics for the controls, low and high power. (The present copper system consists of eight RF units.) The contracts for klystrons, circulators and waveguides have been adjudicated in September 1991, the end of these deliveries is expected by end 1993. Control electronics, RF amplifiers, power meters, temperature stabilised fibre optics for RF reference distribution and associated transmitting equipment have also been ordered in 1991; all the corresponding deliveries have been completed.

The new RF units at Pts 2 and 6 are ready, the four new units at Pt 8 will be completed by this summer, those at Pt 4 will become available during 1994.

A central control of the total 20 RF units, which will be available in 1994, will be ensured by a global RF control system, designed to adjust synchrotron tune and to keep an optimum RF balance; this system is already operational [9] for the presently available accelerating cavities.

\subsection{Cryogenics}

At each of the even LEP Points there is a cryoplant [10] with an initial cooling power of $12 \mathrm{~kW}$ at $4.5 \mathrm{~K}$ and an ultimate one of $18 \mathrm{~kW}$. These cryoplants consist of a surface upper cold box for the $300 \mathrm{~K}$ to $20 \mathrm{~K}$ temperature range, connected by vertical transfer lines in the machine shafts to an underground lower cold box for the $20 \mathrm{~K}$ to $4.5 \mathrm{~K}$ range. The corresponding contracts were adjudicated in December 1990; their execution is progressing close to the contractual planning, i.e. the cryoplants at Pts 6 and 8 have been installed during the last five months and are now being commissioned. They show an inverse efficiency (power from the grid/cooling power at $4.5 \mathrm{~K}$ ) better than $230 \mathrm{~W} / \mathrm{W}$, which is the best ever achieved. The cryoplants at Pts 2 and 4 , at present being installed, will be commissioned by October 1993 and February 1994, respectively.

Two $6 \mathrm{~kW}$ cryoplants have been delivered and are at present routinely operated. One has been installed underground for cooling the SCC's at Pt2 during 1992 and 1993, and the other one equips the SM18 cryogenic test facility, where the acceptance tests of the SC cavities and LHC magnet development work are taking place.

The installation of the $\mathrm{He}$ transfer lines for supplying the SCC's at Pts 2, 6 and 8 has been completed; the installation of those at Pt4 will take place during the next winter shutdown. The delivery of all other components for the cryogenic system (e.g. high and low pressure storage vessels, piping for He gas) will be completed by this summer.

\section{COLLIDER COMPONENTS AND INFRASTRUCTURE}

\subsection{Magnets, separators, vacuum, instrumentation, power converters}

The present SC quadrupoles at the even IP's will be replaced at earliest during the 1993-1994 shut-down by new ones [11], having the same outer overall dimensions, but with a magnetic field gradient increased from 36 to $55 \mathrm{~T} / \mathrm{m}$. A prototype magnet has successfully been tested in November 92 and the series production has started.

The 24 steel-concrete cores of the original injection dipoles saturated as from $70 \mathrm{GeV}$ and have been replaced by classical steel laminated cores during the 92-93 shut-down.

To keep sufficient beam separation during acceleration up to $100 \mathrm{GeV}$, two additional electrostatic separators, identical to those already in operation, will be added at each of the even IP's. The vacuum tanks and the electrodes for these eight new separators have all been delivered, their assembly is taking place at CERN, installation is foreseen for the 94-95 shutdown.

The performance of the vacuum system in presence of the high energy synchrotron radiation has been extrapolated from various measurements [12], and a beam-gas lifetime of about 20 hours is expected. The modifications of the odd and even IP's, and that of the RF straight sections, require that about 130 vacuum chambers for drift spaces and quadrupole magnets be manufactured or modified. The aluminium vacuum chambers are modified at the CERN workshops, whereas the stainless steel chambers have been ordered to industry. The odd IP's and some RF straights at Pt6 (total length about $2 \mathrm{~km}$ ) have been modified during the 92-93 shut-down. The RF cells at Pts 4 and 8 $(700 \mathrm{~m})$, and the even IP's $(240 \mathrm{~m}, 80$ vacuum chambers) will be modified during the 93-94 and 94-95 shutdowns. In total some $17 \mathrm{~km}$ of the LEP vacuum system will have been opened to air and baked out again. The cooling of the vacuum system is likely to be upgraded during the 94-95 shutdown; it will then cope with up to $32 \mathrm{MW}$ of synchrotron power, i.e. $1.6 \mathrm{~kW} / \mathrm{m}$ (electron and positron beams of $6 \mathrm{~mA}$ each at $95 \mathrm{GeV}$ will radiate about $28 \mathrm{MW}$ of synchrotron power). This cooling upgrade will be achieved by changing from series to parallel the water flow in the cooling channels of the vacuum chambers. The lead shielding along the beam 
path will be completed at about 2700 locations also during the 94-95 shutdown.

The major modifications to the beam instrumentation equipment concern the addition or the replacement of collimators[13], to cope with the higher energy deposition by synchrotron radiation. Out of the sixteen collimators in the arcs, shielding the even IP's from off-momentum particles (the absorbing material has changed from aluminium to tungsten) eight are already installed at positions compatible with the $90^{\circ}$ optics. To shield the SC cavities from synchrotron radiation, sixteen new collimators will be installed during the 93-94 shutdown at the outer ends of the RF straight sections.

The original magnet power converters (PC's) were designed for operation up to $65 \mathrm{GeV}$; those for LEP 200 are foreseen for a maximum beam energy of $100 \mathrm{GeV}$. For the LEP bending magnets, the new PC's $(2 \times 9.7 \mathrm{MW})$ have been delivered in October 1991. Because of difficulties with the new large thyristor units, their installation has eventually taken place in the 92-93 shut-down. All the magnet PC's are now ready in their LEP200 configuration; their total number has increased from 758 to 868 units and the installed power from 18 to $50 \mathrm{MW}$. Ten new klystron power converters (40 A, 100 $\mathrm{kV}$ ), identical to those already in use for the present RF system, are necessary. The corresponding contracts were placed in March 1991, their delivery was completed this March.

\subsection{Power distribution, cooling, civil engineering}

The power distribution system required major extensions at $\mathrm{Pt} 2$, where the main dipole PC's are located, and at Pts 4 and 8 , where the new RF units are situated (4 $\times 4.2 \mathrm{MW}$ at each point). The cryoplants need about $3 \mathrm{MW}$ from the grid at each of the even points, where the power converters $(2 \times 1.1 \mathrm{MW})$ for the arc quadrupoles are also situated. Additional power is also necessary for all the other magnet PC's, for the new RF units at Points 2 and 6, for cooling and ventilation and other services. To cope with these requirements, 50 MVA power links ( $10 \mathrm{~km}$ of trenches) from the Prévessin $400 \mathrm{kV} / 66 \mathrm{kV}$ station (where a second $400 \mathrm{kV} / 66 \mathrm{kV}, 110$ MVA transformer has been installed) to Points 4 and 8 have been realised in 1991 . Extensions of the $66 \mathrm{kV}$ substations at Points 2, 4 and 8 with new 38 MVA transformers are operational since this April. All contracts for power distribution equipment, harmonic filters, reactive power compensation, HV and LV switchgear have been completed. To cope with the increase in power dissipation, the number of cooling towers has been increased from four to six at Pts 2 and 6, and from one to five at Pts 4 and 8 (Pts 2 and 6 need additional cooling capacity because of the presence of the copper $R F$ system and resistive experimental magnets). Major extensions of the air and water cooling facilities are needed at Points 4 and 8 , because of the new RF units. All the activities related to the cooling system will be finished by June 1993.

New klystron galleries $(2 \times 2 \times 230 \mathrm{~m})$ have been excavated at Pts 4 and 8 for housing the RF units. At Point 8 , the UA83 and UA87 galleries are completely equipped. At $\mathrm{Pt} 4$ one gallery has been delivered for installation early May, the second one will follow by early June 93 . The five new sound-proofed compressor buildings $\left(5 \times 600 \mathrm{~m}^{2}\right)$ are in service. At Pts 4 and 8 the extensions of the rectifier halls $\left(2 \times 300 \mathrm{~m}^{2}\right)$ and the water pump stations $\left(2 \times 170 \mathrm{~m}^{2}\right)$ have also been delivered to schedule and are already equipped. The twelve new cooling towers described above have been made out of concrete, as are the original ones, and were delivered by December 92 .

\section{SUMMARY}

A high energy optics for LEP is well in hand. The operation of three prototype SCC modules in LEP during 1991 has allowed to gain precious experience, necessary for improving hardware and operational procedures. Concerning SCC's from industry, the transfer of know-how and the begin of series production have required about two years, during which development of power and HOM couplers has been necessary in order to cope with the anticipated higher beam intensities of up to $1 \mathrm{~mA} /$ bunch $\times 8$ bunches/beam [14]. All activities for machine components and infrastructure are in regular progress.

LEP operation at beam energies above the $W$ pair production threshold is foreseen in 1995.

\section{ACKNOWLEDGEMENTS}

In such a short article it is unfortunately not possible to describe or even mention all the Project activities. The ingenuity, determination and untiring efforts of all those contributing to the success of the LEP Energy Upgrade Programme are gratefully acknowledged.

\section{REFERENCES}

[1] LEP Design Report, CERN-LEP/84-01

[2] E. Picasso "Development of RF Superconducting Cavities for Large Storage Rings" Proc. 20th Int.Conf. on High Energy Physics, Madison, 1980.

[3] Ph. Bernard et al "Upgrading of LEP energies by superconducting cavities", CERN/LEP-DI/86-29

[4] C.Wyss "The LEP Energy Upgrade Programme", Particle Accelerator Conference, San Franscsco 1991

[5] R.Bailey et al "LEP Operation in 1992 with a $90^{\circ}$ Optics", this Conference

[6] R.Bailey et al "Commissioning and Operation of the LEP Pretzel Scheme", this Conference

[7] D.Brandt "Lattice Modifications for the LEP Energy Upgrade", EPAC 1992, Vol .1, pp. 403-405

[8] G.Cavallari et al "Superconducting Cavities for the LEP200 Energy Upgrade", this Conference

[9] E.Ciapala et al,"Global Voltage Control for the LEP RF System", this Conference

[10] M. Barranco-Luque et al "Status of Cryogenics for the LEP200 Project", ICEC14, Kiev, June 92

[11] T.Taylor et al 'Design of the Superconducting Quadrupoles for the LEP200 Low-beta Insertions", 12th Intern. Conf. on Magnet Technology, Leningrad, 1991

[12] J-P.Bojon et al "Experience with the Operation of the LEP Vacuum System and its Performance for LEP200", EPAC92, Vol. 2, pp.1564-1566

[13] R.Valbuena et al "Design of a New Generation of Collimators for LEP200", this Conference

[14] L. Evans "LEP Status and Future Plans", this Conference 